\title{
Efficacy of high-versus moderate-dose statin therapy on lower extremity artery disease after revascularization
}

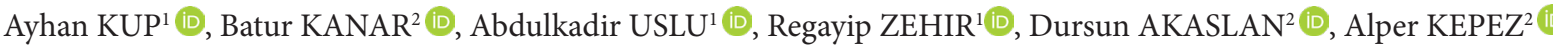 \\ ${ }^{1}$ Cardiology Clinic, Kartal Kosuyolu Heart and Vascular Diseases Research and Training Hospital, Istanbul, Turkey. \\ ${ }^{2}$ Department of Cardiology, School of Medicine, Marmara University, Istanbul, Turkey.
}

Corresponding Author: Batur KANAR

E-mail: baturkanar@hotmail.com

Submitted: 13.07.2021 Accepted: 03.10.2021

\begin{abstract}
Aim: Statins are one of the most important agents in the treatment of atherosclerotic peripheral arterial disease. We aim to compare high - and moderate-dose statin therapy in patients with lower extremity artery disease (LEAD) who have undergone percutaneous transluminal angioplasty (PTA).

Patients and Methods: Ninety-four patients treated with PTA were selected consecutively and retrospectively and were divided into two groups according to the high - or moderate-dose statin treatments they were given. Groups were compared for the absence of restenosis and occlusion as primary patency and the need for reintervention in the treated arterial segment as secondary patency. All patients underwent computed tomography (CT) angiography or duplex scan one year after receiving PTA.

Results: Coronary revascularization $(\mathrm{p}<0.001)$ and prior statin usage $(\mathrm{p}: 0.02)$ were more common in the high-dose statin group when compared to the moderate-dose statin group. Lesion characteristics did not differ between the two groups. Primary patency rate was significantly higher $(36.1 \%$ vs. $27.6 \%$, p 0.01$)$ in the high-dose statin group. Major amputation (4.2\% vs. 10.6\%, p:0.03) and minor amputation (12.7\% vs. $19.1 \%$, p:0.03) rates were significantly lower in the high-dose statin group. Regression analysis revealed that the usage of high-dose statin therapy was an independent predictor of higher primary patency in patients who were treated with prior PTA(Odds ratio:2.208, $\mathrm{p}<0.001$ )

Conclusion: High-dose long-term statin treatment might have better outcomes on primary patency in patients who underwent prior PTA for infrapopliteal lesions as a subgroup of peripheral artery disease. The administration of the high-dose long-term statin therapy might be important in the prognosis of peripheral arterial disease, especially for those with infrapopliteal lesions.

Keywords: Statin treatment, Percutaneous transluminal angioplasty, Peripheral artery disease
\end{abstract}

\section{INTRODUCTION}

Statins are competitive inhibitors of hydroxymethylglutaryl (HMG) CoA reductase, the rate-limiting step in cholesterol biosynthesis. They occupy a portion of the binding site of HMG CoA, blocking access of this substrate to the active site on the enzyme [1]. Statins are one of the main agents in cardiovascular disease in primary and secondary prevention [2]. In addition to coronary heart disease, peripheral artery disease and cerebrovascular events play a major role in secondary prevention. According to the 2019 European Society of Cardiology (ESC) / European Atherosclerosis Society (EAS) guidelines for the management of dyslipidaemias and the lipid modification to reduce cardiovascular risk, intensive statin therapy is recommended especially in patients with clinical atherosclerotic disease, under the age of 75 years old and with atherosclerotic cardiovascular disease (ASCVD) risk>7.5\% [3].

In observational studies and limited randomized clinical trials (RCTs) in patients with lower extremity artery disease (LEAD) (from asymptomatic to severe cases), statin therapy has been shown to cause reductions in all-cause mortality and in cardiovascular (CV) events [4-6]. A systematic review

How to cite this article: Kup A, Kanar B, Uslu A, Zehir R, Akaslan D, Kepez A. Efficacy of high-versus moderate-dose statin therapy on lower extremity artery disease after revascularization. Marmara Med J 2022; 35(1):36-41. doi: 10.5472/marumj.1056187 
of 18 trials including 10000 patients with cholesterol levels ranging from normal to elevated has reported that lipidlowering therapy in subjects affected by atherosclerosis of the lower limbs is associated with a $20 \%$ reduction in total CV events, together with a non-significant $14 \%$ reduction in allcause mortality. Regarding limb prognosis, in the Reduction of Atherothrombosis for Continued Health (REACH) registry, statin use was associated with an $18 \%$ lower rate of adverse limb outcome [4,7].

Infrapopliteal occlusive disease (IP) is a severe and diffuse form of peripheral artery disease (PAD) and requires challenging revascularization procedures. Patients suffering from severe lifestyle-limiting claudication or critical limb ischemia due to atherosclerosis of the crural arteries are often frailer and have higher amputation and mortality rates [8]. In stenotic lesions and short occlusions, endovascular therapy can be the first choice. In long occlusions of crural arteries, bypass with an autologous vein gives superior long-term patency and leg survival. If the patient has increased risk regarding surgery or does not have an autologous vein, endovascular therapy may be attempted [9].

It is important that patients with infrapopliteal lesions receive the best medical treatment (such as lifestyle modification, cessation of smoking, pharmaceutical treatment) as in all atherosclerotic diseases after revascularization [2]. As already mentioned, high doses of statin should be used in patients with clinical atherosclerotic disease. In this study, we aimed to investigate whether there is any difference between vascular patency between two groups receiving high-dose statin and moderatedose statin in a 1-year follow-up of infrapopliteal lesions after revascularization.

\section{PATIENTS and METHODS}

This study was performed retrospectively. All patients had been primarily treated with percutaneous transluminal angioplasty (PTA) of at least one infrapopliteal artery causing severe symptoms or critical limb ischemia (CLI) (Rutherford category 1-6). The indication of PTA was determined based on the 2017 ESC guidelines on the diagnosis and treatment of peripheral arterial diseases [10]. A total 126 limbs of 114 patients were consecutively evaluated. Significant stenosis of inflow arteries if present had to be treated successfully prior to enrollment. The success rate was $92.9 \%$ (106 patients) and technically successful angioplasty of de novo lesions were included. CLI is defined as the presence of chronic ischemic rest pain, ulcers, or gangrene attributable to objectively proven IP [11].

Technical success of infrapopliteal PTA was described as recanalization with antegrade flow and $30 \%$ or less residual stenosis below the knee artery. PTA was performed through anterograde (ipsilateral or contralateral) or retrograde approach at the discretion of the treating interventional cardiologist. Stents were implanted if flow-limiting complications occurred. Unless contraindicated, all patients received aspirin, clopidogrel, high-dose statin (80mg atorvastatin), and cilostazol for at least 1 month after successful recanalization of the vessel. According to the 2019 ESC/EAS guidelines for the management of dyslipidaemias, high-intensity statins received strong Class I recommendations for patients with clinical cardiovascular disease. High-intensity statins are defined as atorvastatin dose of 40-80 mg, and rosuvastatin dose of 20-40 mg. Moderate intensity statins are defined as atorvastatin dose of $10-20 \mathrm{mg}$, and rosuvastatin dose of $10 \mathrm{mg}$ [3]. At follow-up, the patients' statin therapy was determined based on 2019 ESC/EAS guidelines for the management of dyslipidaemias guidelines after the PTA [3].

A total number of 126 limbs in 114 patients who underwent PTA were initially reviewed. Twenty patients $(17.5 \%)$ were excluded from the study. 9 patients were excluded since they were not on statin therapy $(n=9,45 \%), 6$ patients were excluded because they were not on high-dose statin therapy $(n=6,20 \%)$, and 5 patients were excluded for not following up $(n=5,25 \%)$. The remaining eligible patients were divided into two groups according to the statin dose as high - and moderate-dose. Patients were evaluated for primary patency, secondary patency, major and minor amputations and these were identified as clinical outcome. All patients underwent CT angiography or duplex scan a year after the PTA. Loss of patency is defined as restenosis (>50\%) or occlusion of the treated artery was detected by CT angiography or duplex scan. Primary patency was defined as the absence of restenosis, occlusion, or need for re-intervention in the treated arterial segment. Secondary patency was defined as utilizing secondary endoluminal procedures, which involved recanalizing occluded arterial segments. Major amputation was defined as limb loss above the metatarsal level, whereas minor amputation referred to trans metatarsal amputation or removal of more distal parts of the lower extremity.

The present study complies with the principles outlined in the Declaration of Helsinki. The study was approved by the Ethics Committee of Kartal Koşuyolu Heart and Vascular Diseases Research and Training Hospital (protocol number: 2019.7/18234), and written informed consent was obtained from all participated patients.

\section{Statistical Analysis}

All statistical analyses were investigated using a software program (SPSS 22.0 for Mac; SPSS Inc., Chicago, IL, USA). The variables were determined using visual (histograms and probability plots) and analytic methods (Kolmogorov-Smirnov and Shapiro-Wilk tests) to assign whether or not they are normally distributed. Categorical variables are presented as numbers and percentages. Continuous variables are expressed as mean \pm standard deviation. Since all continuous variables were normally distributed, statistical comparisons of quantitative data were performed by an unpaired sample t-test. Logistic regression analyses were performed to determine the predictors of higher primary patency after one year PTA. A p-value of $<0.05$ was considered statistically significant.

\section{RESULTS}

After the exclusions, we identified 94 patients (77[78.7\%] male, 61.7 \pm 8.4 ) who underwent PTA during the study period. Patients were divided into two groups according to the dose of 
statin treatment they received. The first group received a highdose statin therapy (47 patients), and the second group received a moderate-dose statin therapy (47 patients).

Clinical characteristics of patients and lesion features of the two groups were summarized in Table I. Arrhythmia ( $p=0.03)$, coronary artery disease $(\mathrm{p}=0.01)$, coronary revascularization with CABG or PCI $(\mathrm{p}<0.001)$ and prior statin usage $(\mathrm{p}: 0.02)$ were more common in the high-dose statin group. Lesion characteristics did not differ between the groups. There was a statistically significant difference in the clinical outcomes between the two groups. Primary patency rate was significantly higher $(36.1 \%$ vs. $27.6 \%$, p 0.01$)$ in the high-dose statin group. Major amputation (4.2\% vs. $10.6 \%$, p:0.03) and minor amputation $(12.7 \%$ vs. $19.1 \%$, p:0.03) rates were significantly lower in the high-dose statin group. Clinical outcomes were summarized in Table II.

Univariate logistic regression analysis was performed to determine the independent predictors of higher primary patency after one-year PTA (Table III). The use of high-dose statin therapy was found to be an independent predictor of higher primary patency in patients who had received prior PTA (Odds Ratio: 2.208; 95\% confidence interval:1.348-3.899; $\mathrm{p}<0.001)$.

Table I. Clinical characteristics of patients and lesion features with respect to high dose statin usage

\begin{tabular}{|c|c|c|c|}
\hline Characteristics & $\begin{array}{l}\text { High-dose statin group } \\
\qquad(\mathrm{n}=47)\end{array}$ & $\begin{array}{l}\text { Moderate-dose statin group } \\
\qquad(\mathrm{n}=47)\end{array}$ & P value \\
\hline Age, years & $61.3 \pm 8.1$ & $62.1 \pm 8.6$ & 0.72 \\
\hline Male sex, $\mathrm{n}(\%)$ & $37(78.7 \%)$ & $40(85.1 \%)$ & 0.16 \\
\hline Diabetes, $\mathrm{n}(\%)$ & $42(89.3 \%)$ & $41(8 \%)$ & 0.53 \\
\hline Arrhythmia, n (\%) & $14(29.7 \%)$ & $8(17.7 \%)$ & 0.03 \\
\hline Congestive heart failure, $\mathrm{n}(\%)$ & $16(34 \%)$ & $14(29.7 \%)$ & 0.24 \\
\hline Coronary artery disease, $\mathrm{n}(\%)$ & $38(80.8 \%)$ & $20(42.5 \%)$ & 0.01 \\
\hline CABG or PCI, $\mathrm{n}(\%)$ & $36(76.9 \%)$ & $13(27.6 \%)$ & $<0.001$ \\
\hline Hypertension, $\mathrm{n}(\%)$ & $41(87.2 \%)$ & $43(91.1 \%)$ & 0.34 \\
\hline Renal insufficiency, n (\%) & $27(57.4 \%)$ & $30(63.8 \%)$ & 0.54 \\
\hline Dialysis, $\mathrm{n}(\%)$ & $12(25.5 \%)$ & $14(29.7 \%)$ & 0.21 \\
\hline Stroke, $\mathrm{n}(\%)$ & $5(0.10 \%)$ & $3(0.06 \%)$ & 0.20 \\
\hline Current smoker, $\mathrm{n}(\%)$ & $42(89.3 \%)$ & $40(85.1 \%)$ & 0.74 \\
\hline Chronic obstructive pulmonary disease, $\mathrm{n}(\%)$ & $21(44.6 \%)$ & $17(36.1 \%)$ & 0.08 \\
\hline Serum total cholesterol $(\mathrm{mg} / \mathrm{dl})$ & $216 \pm 35$ & $234 \pm 56$ & 0.08 \\
\hline Serum LDL (mg/dl) & $128 \pm 23$ & $134 \pm 36$ & 0.09 \\
\hline Prior statin usage $\mathrm{n}(\%)$ & $25(53.1 \%)$ & $17(36.1 \%)$ & 0.02 \\
\hline \multicolumn{4}{|l|}{ Rutherford category, n (\%) } \\
\hline 4. & $17(36.1 \%)$ & $20(42.5 \%)$ & 0.09 \\
\hline 5. & $20(42.5 \%)$ & $19(40.4 \%)$ & \\
\hline 6. & $10((21.2 \%)$ & $8(17 \%)$ & \\
\hline Previous higher level revascularisation, $\mathrm{n}(\%)$ & $13(27.6 \%)$ & $16(34 \%)$ & 0.11 \\
\hline \multicolumn{4}{|l|}{ Target artery, $\mathrm{n}$ (total) } \\
\hline Anterior tibial & $21(75)$ & $28(66)$ & 0.28 \\
\hline Peroneal & $12(75)$ & $11(66)$ & \\
\hline Posterior tibial & $39(75)$ & $22(66)$ & \\
\hline Tibioperoneal trunk & $3(75)$ & $5(66)$ & \\
\hline Lesion length, $\mathrm{cm}$ & $12 \pm 3$ & $11.3 \pm 3$ & 0.41 \\
\hline Baseline stenosis, \% & $79 \pm 13$ & $75 \pm 12$ & 0.21 \\
\hline Total Occlusions, $\mathrm{n}(\%)$ & $38(80.8 \%)$ & $35(74.4 \%)$ & 0.41 \\
\hline Severe Calcification, $\mathrm{n}(\%)$ & $31(65.9 \%)$ & $28(59.5 \%)$ & 0.35 \\
\hline Below the ankle lesion, $\mathrm{n}(\%)$ & $11(23.4 \%)$ & $8(17 \%)$ & 0.09 \\
\hline \multicolumn{4}{|l|}{ TAsc 2 classification } \\
\hline $\mathrm{A}, \mathrm{B}, \mathrm{C}$ & $16(34 \%)$ & $17(36 \%)$ & 0.5 \\
\hline $\mathrm{D}$ & $31(66 \%)$ & $30(64 \%)$ & 0.5 \\
\hline
\end{tabular}

CABG: coronary artery bypass graft, PCI: percutaneous coronary intervention, TASC: trans atlantic inter-society consensus 
Table II. Clinical endpoints after one-year PTA

\begin{tabular}{|l|c|c|c|} 
& $\begin{array}{c}\text { High-dose } \\
\text { statin group } \\
(\mathbf{n}=47)\end{array}$ & $\begin{array}{c}\text { Moderate- } \\
\text { dose statin } \\
\text { group }(\mathbf{n}=\mathbf{4 7})\end{array}$ & P value \\
\hline Primary patency & $17(36.1 \%)$ & $13(27.6 \%)$ & $\mathbf{0 . 0 1}$ \\
\hline Secondary patency & $15(31.9 \%)$ & $18(38.2 \%)$ & 0.07 \\
\hline Major amputation & $2(4.2 \%)$ & $5(10.6 \%)$ & $\mathbf{0 . 0 3}$ \\
\hline Minor amputation & $6(12.7 \%)$ & $9(19.1 \%)$ & $\mathbf{0 . 0 3}$ \\
\hline
\end{tabular}

Bold values indicate statistical significance $p<0.05$

Table III. Univariate logistic regression analysis to determinate the predictors of higher primary patency after one-year PTA

\begin{tabular}{|l|c|c|c|}
\hline & Odds Ratio & $\begin{array}{c}\mathbf{9 5 \%} \\
\text { Confidence } \\
\text { Interval }\end{array}$ & $\mathbf{p}$ \\
\hline $\begin{array}{l}\text { Usage of high-dose statin } \\
\text { therapy }\end{array}$ & 2.208 & $1.348-3.899$ & $<\mathbf{0 . 0 0 1}$ \\
\hline Age & 1.010 & $1.002-1.017$ & 0.029 \\
\hline Male sex & 1.061 & $0.42-2.62$ & 0.59 \\
\hline $\begin{array}{l}\text { Severe calcification at } \\
\text { lesion }\end{array}$ & 1.024 & $1.001-1.169$ & 0.36 \\
\hline Lesion length & 1.008 & $0.97-1.204$ & 0.48 \\
\hline
\end{tabular}

Bold values indicate statistical significance $p<0.05$

\section{DISCUSSION}

The present study evaluated the long term effects of high-dose statin treatment compared to moderate-dose statin therapy in patients with infrapopliteal artery disease who had undergone successful PTA. The findings of our study show that the primary patency rate was significantly higher in the high-dose statin therapy group, while major amputation and minor amputation rates were significantly lower in the high-dose statin group. The use of high-dose statin therapy was an independent predictor of higher primary patency in patients who had received prior PTA.

Although, it is known that high cholesterol concentrations are associated with a higher risk of developing PAD [12], statins are underprescribed in PAD when compared to other well-known atherosclerotic disease processes [13]. The intensity of statin therapy has also been a matter of debate and the effect of highintensity statin therapy on cardiovascular outcomes is not well understood in patients with PAD $[14,15]$. Clinical studies on femoral plaque characteristics have reported the use of highdose statins to predominantly improve plaque composition or cause plaque regression, leading to a more stable phenotype in PAD [6]. Arya et al. showed that amputation mortality rates were lower in the group receiving high-dose statins in patients with PAD. This study showed an almost 30\% reduction in risk of death and $30-40 \%$ reduction in risk of major amputation in high-intensity statin group. In low and moderate-intensity statin group, extremity loss and mortality were also reduced but not as high as in the high-intensity group [16]. Foley et al. revealed that patients with PAD who were on high-dose statin therapy before receiving endovascular intervention showed improved survival and fewer major adverse cardiovascular events compared to patients on moderate-dose statin therapy. However, they did not find any significant difference in the follow-up patency of lower extremity arteries between the 2 groups [17]. Stoekenborek et al. showed that high-dose statin therapy with atorvastatin significantly reduced the incidence of PAD compared with moderate-dose statin therapy with simvastatin [18]. Spring et al. investigated the effect of highdose atorvastatin on endothelial function and local progression of the disease in patients with PAD. However, they did not find any significant difference between the use of high - and moderate-dose statin therapies [19]. As shown in the literature, the use of statin therapy shows improvement in both plaque stabilization and reduces inflamation in patients with a wide range of atherosclerotic diseases. In our study, we found similar results, that a high dose statin therapy was an effective treatment for atherosclerotic lesions. The high-dose statin group showed statistically significant better outcomes in all the variables we tested. Even though in the literature there are limited studies showing no statistically significant improvements with the use of statin therapy, most of the studies come to a similar conclusion that a high-dose statin therapy provides better overall outcome in atherosclerotic diseases $[6,16,18,19]$.

Infrapopliteal occlusive disease (IP) is a severe and diffuse form of $\mathrm{PAD}$ and requires challenging revascularization procedures. Patients suffering from severe lifestyle-limiting claudication or CLI due to atherosclerosis of the crural arteries are often frailer and have higher amputation and mortality rates [8]. Revascularization of infrapopliteal lesions are predominantly treated with endovascular intervention although revascularization options vary depending on the length of the lesion, the presence or absence of an autologous venous graft, and the surgical risk [9]. PTA remained the most widely used method for recanalization of the infrapopliteal lesions but was significantly limited by the high restenosis and occlusion rates [20]. A meta-analysis by Romiti et al of infrapopliteal angioplasty for the treatment of CLI showed 1-year primary and secondary patency rates of $58 \%$ and $68 \%$, respectively [21]. Another study showed primary patency at 1 year of $33 \%$ with secondary patency of $56 \%$ and a limb salvage rate of $75 \%$ after infrapopliteal angioplasty [22].

High-dose statin therapy is of great importance in the prevention of atherosclerotic cardiovascular diseases and in the prevention of disease progression with both reduction of cholesterol levels and pleitrophic effects in patients with PAD.

Statins have great importance in the prevention of atherosclerotic disease progression by lowering cholesterol levels [2]. In addition to achieving a therapeutic decrease in serum cholesterol levels, statin therapy appears to promote other effects that are independent of changes in serum cholesterol. The effect of statins on the endothelial function in humans remains under discussion. Particularly, it is still unclear if the improvement in endothelial function is due to a reduction in LDL-cholesterol or an arterial pleiotropic effect. These pleiotropic effects include attenuation of vascular inflammation, improved endothelial cell function, stabilization of atherosclerotic plaque, decreased 
vascular smooth muscle cell migration and proliferation, and inhibition of platelet aggregation [23]. Silva et al. showed that the pleiotropic effects of high-dose statins may improve outcomes in atherosclerotic renovascular disease [24]. Subramanian et al. showed that high-dose atorvastatin reduces periodontal inflammation, suggesting a newly recognized effect of statins. Given the concomitant changes observed in periodontal and arterial inflammation, these data raise the possibility that a portion of that beneficial impact of statins on atherosclerosis relates to reductions in extra-arterial inflammation [25].

\section{Study limitations}

Our study has several limitations. First, our study was a singlecenter and non-randomized study. Secondly, a relatively small number of the patients were enrolled in our study. Thirdly, the procedures were not performed by a single team, and even though all the operators were experienced interventional cardiologists, revascularization strategy was at the discretion of the operator. Also, the high-dose statin treatment group had lower low-density lipoprotein (LDL) cholesterol levels compared to the moderate-statin treatment group although it was statistically insignificant. However, based on the 2019 ESC/ EAS guidelines for the management of dyslipidaemias guideline, the LDL cholesterol level should be $<70 \mathrm{mg} / \mathrm{dL}$ [3]. This value might affect the findings of our study.

\section{Conclusion}

Statins are of great importance in improving cardiovascular protection, inhibiting the progression of $\mathrm{PAD}$, and improving clinical outcomes after interventional therapy in PAD. Highdose long-term statin treatment might have better outcomes on primary patency in infrapopliteal lesions as a subgroup of PAD after PTA. The administration of the high-dose longterm statin therapy might be important in the prognosis of patients with infrapopliteal lesions. Further multi-centered and randomized clinical studies are needed to clarify the possible causal relationship.

\section{Compliance with the Ethical Standards}

\section{Ethical Approval:}

The present study complies with the principles outlined in the Declaration of Helsinki. The study was approved by the Ethics Committee of Kartal Koşuyolu Heart and Vascular Diseases Research and Training Hospital, Istanbul (protocol number: 2019.7/18-234), and written informed consent was obtained from all participated patients

Financial Support: The authors have no relevant financial information to close.

Conflict of Interest: The authors have no potential conflicts of interest to disclose.

\section{Author contributions:}

All authors contributed to the study conception and design. AK was the primary investigator of the study, AK:Design of the study, data collection, analysis of the data and drafting of the article, BK and AK: Critical revision of the article, AU, RZ, and DA: Analysis and collecting of the data and drafting of the article. All authors read and approved the final version of the article.

\section{REFERENCES}

[1] Istvan ES, Deisenhofer J. Structural mechanism for statin inhibition of HMG-CoA reductase. Science 2001;292:1160-4. doi: 10.1126/science.1059344.

[2] Catapano AL, Graham I, De Backer G, et al. 2016 ESC/EAS Guidelines for the Management of Dyslipidaemias. EurHeart J 2016; 37:2999. doi: 10.1093/eurheartj/ehw272.

[3] Mach F, Baigent C, Catapano AL, et al. ESC Scientific Document Group. 2019 ESC/EAS Guidelines for the management of dyslipidaemias: lipid modification to reduce cardiovascular risk. Eur Heart J 2020 Jan 1; 41:111-88. doi: 10.1093/eurheartj/ehz455.

[4] Aung PP, Maxwell HG, Jepson RG, et al. Lipid-lowering for peripheral arterial disease of the lower limb. Cochrane Database Syst Rev 2007; 4; CD000123. doi:10.1002/14651858. CD000123.pub2.

[5] Antoniou GA, Fisher RK, Georgiadis GS, et al. Statin therapy in lower limb peripheral arterial disease: systematic review and meta-analysis.Vascul Pharmacol 2014;63:79-87. doi: 10.1016/j.vph.2014.09.001.

[6] Heart Protection Study Collaborative Group. Randomized trial of the effects of cholesterol-lowering with simvastatin on peripheral vascular and other major vascular outcomes in 20,536 people with peripheral arterial disease and other highrisk conditions. J Vasc Surg 2007; 45:645-54. doi: 10.1016/j. jvs.2006.12.054.

[7] Kumbhani DJ, Steg PG, Cannon CP, et al. Statin therapy and long-term adverse limb outcomes in patients with peripheral artery disease: insights from the REACH registry. EurHeart J 2014; 35:2864-72. doi: 10.1093/eurheartj/ehu080.

[8] Rand T, Uberoi R. Current status of interventional radiology treatment of infrapopliteal arterial disease. Cardiovasc Intervent Radiol 2013; 36: 588-98. doi: 10.1007/s00270.012.0524-8.

[9] Aboyans V, Ricco JB, Bartelink MEL, et al. 2017 ESC Guidelines on the Diagnosis and Treatment of Peripheral Arterial Diseases, in collaboration with the European Society for Vascular Surgery (ESVS). Rev Esp Cardiol (EnglEd) 2018 ; 71:111. doi: 10.1016/j.rec.2017.12.014.

[10] Aboyans V, Ricco JB, Bartelink MEL, et. Al. ESC Scientific Document Group. 2017 ESC Guidelines on the Diagnosis and Treatment of Peripheral Arterial Diseases, in collaboration with the European Society for Vascular Surgery (ESVS): Document covering atherosclerotic disease of extracranial carotid and vertebral, mesenteric, renal, upper and lower extremity arteriesEndorsed by: the European Stroke Organization (ESO) The Task Force for the Diagnosis and Treatment of Peripheral Arterial Diseases of the European Society of Cardiology (ESC) and of the European Society for Vascular Surgery (ESVS). Eur Heart J 2018 ; 39:763-816. doi: 10.1093/eurheartj/ehx095. 
[11] Elsayed S, Clavijo LC. Critical limb ischemia. Cardiol Clin 2015; 33:37-47. doi: 10.1016/j.ccl.2014.09.008.

[12] Fowkes FG, Housley E, Riemersma RA, et al. Smoking, lipids, glucose intolerance, and blood pressure as risk factors for peripheral atherosclerosis compared with ischemic heart disease in the Edinburgh Artery Study. Am J Epidemiol 1992; 135:331-40. doi: 10.1093/oxfordjournals.aje.a116294

[13] Schmidt A, Ulrich M, Winkler B, et al. Angiographic patency and clinical outcome after balloon-angioplasty for extensive infrapopliteal arterial disease. Catheter Cardiovasc Interv 2010;76:1047-54. doi: 10.1002/ccd.22658.

[14] Cannon CP, Steinberg BA, Murphy SA, et al. Meta-analysis of cardiovascular outcomes trials comparing intensive versus moderate statin therapy. J Am Coll Cardiol 2006;48:438-45. doi: 10.1016/j.jacc.2006.04.070.

[15] Pedersen TR, Faergeman O, Kastelein JJ, et al. Highdose atorvastatin vs usual-dose simvastatin for secondary prevention after myocardial infarction: the IDEAL study: a randomized controlled trial. JAMA 2005;294:2437-45. doi: 10.1001/jama.294.19.2437.

[16] Arya S, Khakharia A, Binney ZO, et al. Association of statin dose with amputation and survival in patients with peripheral artery disease. Circulation 2018;137:1435-46. doi: 10.1161/ CIRCULATIONAHA.117.032361.

[17] Foley TR, Singh GD, Kokkinidis DG, et al. High-intensity statin therapy is associated with improved survival in patients with peripheral artery disease. J Am Heart Assoc 2017; 6:e005699. doi: 10.1161/JAHA.117.005699.

[18] Stoekenbroek RM, Boekholdt SM, Fayyad R, et al. Incremental decrease in end points through aggressive lipid lowering study group. High-dose atorvastatin is superior to moderate-dose simvastatin in preventing peripheral arterial disease. Heart 2015;101:356-62. doi: 10.1136/heartjnl-2014-306906.

[19] Spring S, Simon R, van der Loo B, et al. High-dose atorvastatin in peripheral arterial disease (PAD): effect on endothelial function, intima-media-thickness and local progression of PAD. An open randomized controlled pilot trial. Thromb Haemost 2008 ;99:182-9.

[20] Fowkes FG, Rudan D, Rudan I, et al. Comparison of global estimates of prevalence and risk factors for peripheral artery disease in 2000 and 2010: a systematic review and analysis. Lancet 2013;382:1329-40. doi: 10.1160/TH07-04-0265.

[21] Romiti M, Albers M, Brochado-Neto FC, et al. Meta-analysis of infrapopliteal angioplasty for chronic critical limb ischemia. J Vasc Surg 2008:47:975-81. doi: 10.1016/j.jvs.2008.01.005.

[22] Fernandez N, McEnaney R, Marone LK, et al. Predictors of failure and success of tibial interventions for critical limb ischemia. J Vasc Surg 2010 ;52:834-42. doi: 10.1016/j. jvs.2010.04.070.

[23] Sadowitz B, Maier KG, Gahtan V. Basic science review: Statin therapy-Part I: The pleiotropic effects of statins in cardiovascular disease. Vasc Endovascular Surg 2010 ;44:24151. doi: 10.1177/153.857.4410362922. .

[24] Silva VS, Martin LC, Franco RJ, et al. Pleiotropic effects of statins may improve outcomes in atherosclerotic renovascular disease. Am J Hypertens2008 ;21:1163-8. doi: 10.1038/ ajh.2008.249.

[25] Subramanian S, Emami H, Vucic E, et al. High-dose atorvastatin reduces periodontal inflammation: a novel pleiotropic effect of statins. J Am Coll Cardiol 2013 ;62:238291. doi: 10.1016/j.jacc.2013.08.1627. 